\title{
TERT promoter mutations contribute to subset prognostication of lower-grade gliomas
}

Aden Ka-Yin Chan ${ }^{1,2,5}$, Yu Yao ${ }^{3,5}$, Zhenyu Zhang ${ }^{3}$, Nellie Yuk-Fei Chung ${ }^{1,2}$, Joseph Shu-Ming Liu ${ }^{1,2}$, Kay Ka-Wai Li ${ }^{1,2}$, Zhifeng Shi ${ }^{3}$, Danny Tat-Ming Chan ${ }^{4}$, Wai Sang Poon ${ }^{4}$, Liangfu Zhou ${ }^{3}$ and Ho-Keung Ng ${ }^{1,2}$

${ }^{1}$ Department of Anatomical and Cellular Pathology, The Chinese University of Hong Kong, Hong Kong, China; ${ }^{2}$ The Chinese University of Hong Kong-Shenzhen Research Institute, Shenzhen, China; ${ }^{3}$ Department of Neurosurgery, Huashan Hospital, Fudan University, Shanghai, China and ${ }^{4}$ Neurosurgery Division, Department of Surgery, The Chinese University of Hong Kong, Hong Kong, China

Recurrent mutations in the promoter region of telomerase reverse transcriptase (TERT) have been found in various cancers including diffuse gliomas. Mutations lead to TERT upregulation and are associated with aggressive clinical behavior in glioblastomas. However, the clinical significance of TERT promoter mutations in lower-grade gliomas remains undetermined. The aim of this study is to evaluate the status of TERT promoter and the respective prognostic significance in a cohort of 237 lower-grade gliomas comprising grades II and III astrocytomas, oligodendrogliomas, and oligoastrocytomas. Mutually exclusive mutations in TERT promoter, C228T and C250T, were identified in 16/105 (15\%) diffuse astrocytomas, 16/63 (25\%) anaplastic astrocytomas, $13 / 18(72 \%)$ oligodendrogliomas, $3 / 3(100 \%)$ anaplastic oligodendrogliomas, $17 / 45(38 \%)$ oligoastrocytomas, and $2 / 3(67 \%)$ anaplastic oligoastrocytomas. Mutations co-occurred with $1 p / 19 q$ codeletion $(P<0.001)$ and are associated with oligodendroglial histology $(P<0.001)$. Kaplan-Meier's survival analysis showed that TERT promoter mutation $(P=0.037)$, Isocitrate dehydrogenase $(I D H)$ mutation $(P<0.001)$, and $1 \mathrm{p} / 19 \mathrm{q}$ codeletion $(P<0.001)$ were associated with favorable overall survival $(O S)$. In the subset of 116 IDH-mutated lower-grade gliomas lacking 1p/19q codeletion, 19 TERT promoter-mutated tumors exhibited longer progression-free survival (PFS) $(P=0.027)$ and OS $(P=0.004)$. Consistent with this observation, in the subset of $97 \mathrm{IDH}$-mutated astrocytomas, 14 TERT promoter-mutated tumors showed longer PFS $(P=0.001)$ and OS $(P=0.001)$. In contrast, among the subset of $74 \mathrm{IDH}$ wild-type lower-grade gliomas with intact 1p/19q, TERT promoter mutation was associated with shorter PFS $(P=0.001)$ and OS $(P=0.001)$. Similarly, in the subset of $65 I D H$ wild-type astrocytomas, 16 TERT promoter-mutated tumors exhibited unfavorable PFS $(P=0.007)$ and OS $(P=0.008)$. Our results indicate that when combined with IDH status, TERT promoter mutation contributes to prognostic subgroups of lower-grade astrocytic tumors or $1 p / 19 q$ intact lower-grade gliomas and this may further refine future molecular classification of lower-grade gliomas.

Modern Pathology (2015) 28, 177-186; doi:10.1038/modpathol.2014.94; published online 1 August 2014

Diffuse gliomas, the most common primary malignant brain tumors, are classified by the World Health Organization (WHO) into astrocytoma, oligodendroglioma, and oligoastrocytoma based on

Correspondence: Dr AK-Y Chan or Professor H-K Ng, Department of Anatomical and Cellular Pathology, The Chinese University of Hong Kong, 30-32 Ngan Shing Street, Shatin, Hong Kong, China or Professor L Zhou, Department of Neurosurgery, Huashan Hospital, Fudan University, Wulumuqi Road 12\#, Shanghai 200040, China. E-mail: adenchan@cuhk.edu.hk or hkng@cuhk.edu.hk or lfzhouc@126.com

${ }^{5}$ These authors contributed equally to this work and are co-first authors.

Received 27 January 2014; revised 20 May 2014; accepted 21 May 2014; published online 1 August 2014 histology and further graded into grade II to grade IV according to malignant features. ${ }^{1}$ Lower-grade gliomas, comprising grade II and grade III diffuse gliomas, exhibit an infiltrative nature and intrinsic tendency to recur or progress to higher-grade lesion ie grade IV glioblastoma. Although the current classification of lower-grade gliomas is based on histology and has prognostic implication, heterogeneous clinical outcomes exist among gliomas within each group. Such histology-based classification system also leads interobserver variability in diagnosis. ${ }^{2}$ Mounting evidence has suggested that biomarkers can aid tumor diagnosis, determine prognosis, and guide clinical management. Chromosome $1 p$ and $19 q$ codeletion, the genetic hallmark of 
oligodendrogliomas associated with long survival and chemo-radio sensitivity, represents the prototype molecular marker with unequivocal diagnostic, prognostic, and therapeutic utilities in diffuse gliomas. ${ }^{3-7}$ Isocitrate dehydrogenase (IDH) mutation is probably the most important molecular marker discovered in diffuse gliomas with breakthrough clinical values in the recent years. ${ }^{8}$ Apart from its use in difficult diagnostic situations, ${ }^{8-10}$ mutation of this enzyme also stratifies diffuse gliomas prognostically. ${ }^{11,12}$ Recently, recurrent mutations in the promoter region of telomerase reverse transcriptase (TERT), the gene encoding catalytic subunit of telomerase, were detected in $\sim 70 \%$ of malignant melanomas. ${ }^{13,14}$ Mutations caused a cytosine-tothymine transition at the positions of chr5, 1295228 (C228T) and 1295250 (C250T), and generated an identical 11 base-pair nucleotide sequence $\left(5^{\prime}\right.$-CCC CTTCCGGG-3') containing a consensus binding site $\left(5^{\prime}\right.$-TTCC-3') for E-twenty-six transcription factors. ${ }^{13-15}$ Importantly, this promoter mutation is associated with upregulation of TERT expression, suggesting it as a mechanism of telomerase activation in tumorigenesis. ${ }^{14,16}$ Further studies demonstrated the high frequency of TERT promoter mutation in different histological types of diffuse gliomas, in particular oligodendrogliomas, oligoastrocytomas, and primary glioblastoma. ${ }^{16-18}$ Although TERT promoter mutation was shown to be associated with poor clinical outcome in glioblastoma patients, ${ }^{15,18}$ clinical value of this newly identified mutation in lower-grade gliomas remains elusive. In this study, we conducted mutational analysis for TERT promoter in 237 lower-grade gliomas with the aim to examine the clinical value of TERT promoter mutation in terms of classification and prognostication in lower-grade gliomas.

\section{Materials and methods}

\section{Patients and Tissue Samples}

A total of 237 lower-grade gliomas diagnosed between 1990 and 2012 with formalin-fixed paraffin-embedded tissues available were retrieved from the tissue archive of the Department of Anatomical and Cellular Pathology, Prince of Wales Hospital (Hong Kong) and Department of Neurosurgery, Huashan hospital (Shanghai), comprising 105 diffuse astrocytomas (WHO grade II; AII), 63 anaplastic astrocytomas (WHO grade III; AAIII), 18 oligodendrogliomas (WHO grade II; OII), 3 anaplastic oligodendrogliomas (WHO grade III; AOIII), 45 oligoastrocytomas (WHO grade II; OAII), and 3 anaplastic oligoastrocytomas (WHO grade III; AOAIII). All cases were stained with haematoxylin \& eosin and reviewed according to the 2007 WHO criteria. ${ }^{1}$ Clinical and survival data of the patients were retrieved from the respective institutional medical record systems. This study was approved by the
Ethics Committee of Shanghai Huashan Hospital and the New Territories East Cluster-Chinese University of Hong Kong Ethics Committee.

\section{Mutation Analysis of TERT Promoter and IDH1/IDH2}

Tissues from representative tumor area with tumor content $>70 \%$ were scrapped off from dewaxed sections and treated with proteinase $\mathrm{K}$ at a final concentration of $2 \mu \mathrm{g} / \mu \mathrm{l}$ in $10 \mathrm{mM}$ Tris- $\mathrm{HCl}$ buffer $\left(\mathrm{pH} 8.5\right.$ ) at $55^{\circ} \mathrm{C}$ for $2-18 \mathrm{~h}$ and then at $98^{\circ} \mathrm{C}$ for $10 \mathrm{~min}$. The crude cell lysate was centrifuged and supernatant was used for subsequent PCR analysis. The forward primer TERT-F (5'-GTCCTGCCCCTTCA CCTT-3') and reverse primer TERT-R (5'-CAGCGCTG CCTGAAACTC-3') were used to amplify a $163 \mathrm{bp}$ fragment spanning the two mutational hotspots (chr5, 1295228 (C228T) and 1295250 (C250T)) in TERT promoter region (Figure 1, Supplementary Figure S1 and S2). PCR was performed in $10 \mu \mathrm{l}$ reaction mixture containing $1 \mu \mathrm{l}$ of cell lysate, $0.3 \mathrm{mM}$ of each dNTP, $2.5 \mathrm{mM} \mathrm{MgCl}_{2}, 0.3 \mu \mathrm{M}$ of each primer, and $0.2 \mathrm{U}$ of KAPA HiFi HotStart DNA Polymerase (Kapa Biosystems Wilmington, DE, USA), and was initiated at $95^{\circ} \mathrm{C}$ for $5 \mathrm{~min}$, followed by $40-45$ cycles of $98{ }^{\circ} \mathrm{C}$ for $20 \mathrm{~s}, 68^{\circ} \mathrm{C}$ for $15 \mathrm{~s}$ and $72{ }^{\circ} \mathrm{C}$ for $30 \mathrm{~s}$, and a final extension of $72{ }^{\circ} \mathrm{C}$ for 1 min. Products were then treated with exonuclease I and alkaline phosphatase (TakaRa, Japan). Sequencing was performed using BigDye Terminator Cycle Sequencing kit v1.1 (Life Technologies). The products were resolved in Genetic Analyzer 3130xl and analyzed by Sequencing Analysis software. Mutational hotspots of $I D H 1$ at R132 and IDH2 at R172 were evaluated by direct sequencing as previously described. ${ }^{19}$

\section{Chromosome 1p/19q Status by Fluorescence in situ Hybridization}

Chromosome $1 \mathrm{p} / 19 \mathrm{q}$ status was evaluated by fluorescence in situ hybridization as reported previously. $^{20}$ In brief, 5- $\mu \mathrm{m}$ thick formalin-fixed, paraffin-embedded sections were deparaffinized in xylene, treated with $1 \mathrm{M}$ sodium thiocyanate at $80^{\circ} \mathrm{C}$ for $10 \mathrm{~min}$, digested in pepsin solution at $37^{\circ} \mathrm{C}$ for 20-30 min, rinsed in milli-Q water and dehydrated.

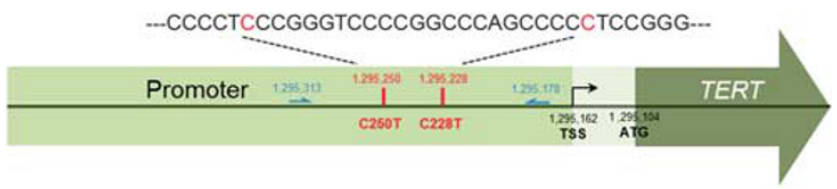

Figure 1 Schematic diagram showing the positions of the two mutation hotspots (red), the primers for PCR (blue), the transcription start site (TSS) (black) and translation start site (ATG) (black) in the promoter region of TERT. Numbers denote the nucleotide positions on chromosome 5 . The sequence at the top shows the wild-type sequence of the promoter region spanning the two hotspots (red). 
Locus-specific probes were generated from bacterial artificial chromosome clones using nick translation, in the presence of Spectrum Orange deoxyuridine triphosphate (dUTP) or Spectrum Green dUTP. The labeled probes were mixed with Cot-1 DNA (Life Technologies) in Hybrisol VI solution (Appligene Oncor, Graffenstaden, France), applied to the section and denatured. Hybridization was carried out at $37^{\circ} \mathrm{C}$ overnight for $16 \mathrm{~h}$. Sections were washed in $1.5 \mathrm{M}$ urea in $0.1 \times$ saline sodium citrate at $48^{\circ} \mathrm{C}$ for $30 \mathrm{~min}$ and then in $2 \times$ saline sodium citrate at $48^{\circ} \mathrm{C}$ for $5 \mathrm{~min}$. After washing, sections were stained with Vectashield mounting medium containing 4',6-diamidino-2-phenylindole (Vector Laboratories) and viewed under a Zeiss Axioplan fluorescence microscope (Carl Zeiss Microscopy LLC, NY, USA). Hybridizing signals in at least 100 non-overlapping nuclei were counted. The loci interrogated were 1p36.3 (RP11-62M23 labeled red)/1q25.3-q31.1 (RP11-162L13 labeled green) and 19q13.3 (CTD2571L23 labeled red)/19p12 (RP11-420K14 labeled green). A sample was considered $1 p$ or $19 q$ deleted when $>50 \%$ of counted nuclei exhibited one target (red) signal and two reference (green) signals.

\section{Statistical Analysis}

Statistical analysis was performed using IBM SPSS Statistics 20 (IBM Corporation, NY, USA). Correlation between molecular markers and clinical parameters were examined by $\chi^{2}$-test or Fisher's exact test, whichever was appropriate. Comparison between two groups was performed by Student's t-test or Mann-Whitney $U$-test. Comparison between three or more groups used one-way analysis of variance (ANOVA) and post hoc analysis with Bonferroni correction. Overall survival (OS) was defined as the time between diagnosis and death or last follow-up. Progression-free survival (PFS) was defined as the time between diagnosis and tumor recurrence or progression. Survival curves were plotted by Kaplan-Meier method and analyzed by Log-rank test. Multivariate analysis was performed by Cox proportional hazards model. $P$-value of $<0.05$ (two sided) was considered statistically significant.

\section{Results}

\section{Cohort Characteristic}

The male to female ratio of the cohort was 1:0.63. The mean and median age of the patients were 40.5 years and 40 years (range 3-9), respectively. There were 15 pediatric patients (at or below 18 years) (five diffuse astrocytomas, five anaplastic astrocytomas, one oligodendroglioma and four oligoastrocytomas) in the cohort. Among the 224 cases with known tumor location, 89 (40\%), 49 (22\%), $25(11 \%)$, and 3 $(1 \%)$ tumors located in frontal, temporal, parietal, and occipital lobe, respectively. Twelve (5\%) cases involved infratentorial region, 24 (11\%) cases involved more than one lobe and $22(10 \%)$ cases involved other regions including corpus callosum, thalamus, and basal ganglia. Treatment data in operation, radiotherapy, and chemotherapy was available in 214 of 237 (90\%), 201 of 237 (85\%), and 200 of 237 $(84 \%)$ patients, respectively. In total,123 of 214 $(58 \%)$ patients received total resection, 145 of 201 $(72 \%)$ patients received radiotherapy and 114 of 200 $(57 \%)$ patients received chemotherapy. In total, 101 of $200(51 \%)$ patients received concomitant chemoradiotherapy.

\section{TERT Promoter Mutation}

Mutation in TERT promoter was found in 67 of 237 (28\%) lower-grade gliomas examined, including 16 of $105(15 \%)$ diffuse astrocytomas, 16 of $63(25 \%)$ anaplastic astrocytomas, 13 of $18(72 \%)$ oligodendrogliomas, 3 of $3(100 \%)$ anaplastic oligodendrogliomas, 17 of 45 (38\%) oligoastrocytomas and, 2 of $3(67 \%)$ anaplastic oligoastrocytomas. Two pediatric astrocytomas (one diffuse astrocytoma and one anaplastic astrocytoma) harbored TERT promoter mutation. Among the 67 mutated tumors, C228T and C250T mutations were mutually exclusive and were observed in $44(19 \%)$ cases (12/105 diffuse astrocytomas, 12/63 anaplastic astrocytomas, 7/18 oligodendrogliomas, 2/3 anaplastic oligodendrogliomas, $10 / 45$ oligoastrocytomas and $1 / 3$ anaplastic oligoastrocytomas) and $23(10 \%)$ cases (4/105 diffuse astrocytomas, 4/63 anaplastic astrocytomas, 6/18 oligodendrogliomas, 1/3 anaplastic oligodendrogliomas, 7/45 oligoastrocytomas and 1/3 anaplastic oligoastrocytomas), respectively (Table 1, Figure 2a and Supplementary Figure S2).

Correlating TERT promoter mutation with clinicopathological variables, patients with TERT promoter mutation were older than those without the mutation (mean age 44.3 vs $39, P=0.007$ ). No association was observed between TERT promoter mutation and gender. Tumors with oligodendroglial histology showed high frequency of TERT promoter mutation (16/21, 76\%) compared with tumors with mixed oligoastrocytic histology $(19 / 48,40 \%)$ and tumors with astrocytic histology $(32 / 168,19 \%)$ $(P<0.001)$ (Figure 2b).

Forty-nine of $67(73 \%)$ TERT-mutated tumors harbored IDH mutation and 109 of $170(64 \%)$ TERT wild-type tumors had $I D H$ mutation (Figure 2c). Among the IDH-mutated tumors, TERT promoter mutation was found in 14/98 (14\%), 16/20 (80\%), and 19/40 (48\%) gliomas with astrocytic, oligodendroglial, and oligoastrocytic histology, respectively $(P<0.001)$. Among tumors with wild-type $I D H$, TERT promoter mutation was detected in 18/70 $(22 \%)$ gliomas with astrocytic histology exclusively (6 diffuse astrocytomas and 12 anaplastic astrocytomas). Wild-type TERT was found in 52/70 (74\%), 1/1, and $8 / 8$ tumors with astrocytic, oligodendroglial, 
Table 1 Summary of clinical and molecular data of lower-grade gliomas in the study

\begin{tabular}{|c|c|c|c|c|c|c|c|c|c|c|c|c|}
\hline \multirow[b]{2}{*}{ Diagnosis } & \multirow[b]{2}{*}{$\mathrm{N}$} & \multirow[b]{2}{*}{ Male } & \multirow[b]{2}{*}{$\begin{array}{c}\text { Mean } \\
\text { age } \\
\text { (years) }\end{array}$} & \multirow[b]{2}{*}{$\begin{array}{c}\text { Median } \\
\text { PFS } \\
\text { (months) }\end{array}$} & \multirow[b]{2}{*}{$\begin{array}{c}\text { Median } \\
\text { OS } \\
\text { (months) }\end{array}$} & \multicolumn{3}{|c|}{ TERT promoter mutation } & \multicolumn{3}{|c|}{ IDH mutation } & \multirow[b]{2}{*}{$\begin{array}{c}1 p / 19 q \\
\text { codeletion } \\
(\%)\end{array}$} \\
\hline & & & & & & C228T & $C 250 T$ & $\begin{array}{c}\text { Combined } \\
\%\end{array}$ & $I D H 1$ & IDH2 & $\begin{array}{c}\text { Combined } \\
\%\end{array}$ & \\
\hline Diffuse astrocytoma & 105 & $60 \%$ & 39.9 & 65.3 & 101.2 & 12 & 4 & $15 \%$ & 69 & 2 & $68 \%$ & $7 \%$ \\
\hline Anaplastic astrocytoma & 63 & $76 \%$ & 43.3 & 16 & 22 & 12 & 4 & $25 \%$ & 26 & 1 & $43 \%$ & $0 \%$ \\
\hline Oligodendroglioma & 18 & $44 \%$ & 41.2 & 108 & NR & 7 & 6 & $72 \%$ & 17 & 0 & $94 \%$ & $59 \%$ \\
\hline $\begin{array}{l}\text { Anaplastic } \\
\text { oligodendroglioma }\end{array}$ & 3 & $33 \%$ & 50.7 & 24 & 24 & 2 & 1 & $100 \%$ & 3 & 0 & $100 \%$ & $67 \%$ \\
\hline Oligoastrocytoma & 45 & $51 \%$ & 37.2 & NR & 120.6 & 10 & 7 & $38 \%$ & 34 & 3 & $82 \%$ & $41 \%$ \\
\hline Anaplastic oligoastrocytoma & 3 & $67 \%$ & 38 & 19.6 & 37.2 & 1 & 1 & $67 \%$ & 2 & 1 & $100 \%$ & $0 \%$ \\
\hline
\end{tabular}

Abbreviations: N, number of case; NR, median survival not yet reached; OS, overall survival; PFS, progression-free survival.

and oligoastrocytic histology, respectively. Opposite correlations between IDH mutation and TERT promoter mutation were observed in subsets of astrocytic tumors with intact $1 \mathrm{p} / 19 \mathrm{q}$ and oligodendroglial tumors. Among the astrocytic tumors with intact $1 \mathrm{p} / 19 \mathrm{q}, 9$ of $27(33 \%)$ TERT-mutated tumors harbored $I D H$ mutation and 80 of $131(61 \%)$ TERT wild-type tumors had IDH mutation $(P=0.008)$ (Figure 2d). In contrast, among the subsets of oligodendroglial tumors, all 35 TERT-mutated tumors harbored $I D H$ mutation and 25 of 34 (74\%) TERT wild-type tumors had IDH mutation $(P=0.001)$ (Figure 2e). Notably, Patients with $I D H$ wild-type-TERT-mutated tumors were older (mean age $=51$ years) than those with $I D H$ wild-type-TERT wild-type tumors (mean age $=36.3$ years, $P<0.001$ ), $I D H$-mutated-TERT wild-type tumors (mean age $=$ 40.6 years, $P=0.012$ ) and trended to be older than $I D H$-mutated-TERT-mutated tumors (mean age $=$ 41.8 years, $P=0.068$ ) (Figure $2 \mathrm{~g}$ ).

Chromosome 1p/19q codeletion was detected in 28 of $65(43 \%)$ TERT-mutated tumors and 9 of 167 (5\%) TERT wild-type tumors $(P<0.001)$ (Figure 2f). Among the 1p/19q codeleted tumors, TERT promoter mutation was identified in $5 / 7(71 \%)$ gliomas with astrocytic histology, 10/12 (83\%) gliomas with oligodendroglial histology, and 13/18 (72\%) gliomas with oligoastrocytic histology. Among tumors lacking $1 \mathrm{p} / 19 \mathrm{q}$ codeletion, $27 / 158(17 \%)$ gliomas with astrocytic histology, 5/8 (63\%) gliomas with oligodendroglial histology and 5/29 (17\%) gliomas with oligoastrocytic histology harbored TERT promoter mutation $(P=0.006)$.

\section{1p/19q Codeletion}

Chromosome 1p/19q codeletion was detected in $37 / 232(16 \%)$ cases including 7/102 (7\%) diffuse astrocytomas, 10/17 (59\%) oligodendrogliomas, 2/3 $(67 \%)$ anaplastic oligodendrogliomas, and 18/44 (41\%) oligoastrocytomas (Table 1, Figure 2a). Five cases (three diffuse astrocytomas, one oligodendroglioma and one oligoastrocytoma) were not examined for $1 \mathrm{p} / 19 \mathrm{q}$ FISH owing to the lack of tissue section. None of the 15 pediatric gliomas had 1p/19q codeletion. All $371 \mathrm{p} / 19 \mathrm{q}$ codeleted tumors harbored IDH mutation and 117/195 (60\%) tumors lacking $1 \mathrm{p} / 19 \mathrm{q}$ codeletion harbored $\mathrm{IDH}$ mutation $(P<0.001)$.

\section{IDH Mutation}

IDH mutation was found in 71/105 (68\%) diffuse astrocytomas, 27/63 (43\%) anaplastic astrocytomas, $17 / 18(94 \%)$ oligodendrogliomas, $3 / 3(100 \%)$ anaplastic oligodendrogliomas, 37/45 (82\%) oligoastrocytomas, and $3 / 3(100 \%)$ anaplastic oligoastrocytomas, with an overall mutation frequency of $67 \%$ (158/237) (Table 1, Figure 2a). In the 158 $I D H$-mutated gliomas, 151 tumors harbored IDH1 mutation and 7 tumors harbored IDH2 mutation. One 18-year-old patient with anaplastic astrocytoma had mutation in IDH1.

\section{Survival Analysis}

Follow-up data were available in 231 patients. The median follow-up, median PFS and median OS of the cohort were 113 months, 56 months, and 83.2 months, respectively.

Univariate analysis showed age $\leq 35$ years $(P=0.02)$, WHO grade II $(P<0.001)$, oligodendroglial histology $(P=0.006), I D H$ mutation $(P<0.001)$, and $1 \mathrm{p} / 19 \mathrm{q}$ codeletion $(P<0.001)$ were associated with longer PFS. Age $\leq 35$ years $(P=0.005)$, WHO grade II $(P<0.001)$, oligodendroglial histology $(P<0.001)$, TERT promoter mutation $(P=0.037)$, IDH mutation $(P<0.001)$, and $1 \mathrm{p} / 19 \mathrm{q}$ codeletion $(P<0.001)$ were associated with longer OS (Table 2, Figure 3a-f). Prognostic value of TERT promoter mutation was further evaluated in subset analysis (Table 3, Figure 3g-r). Among the $157 \mathrm{IDH}$-mutated lower-grade gliomas, 49 TERT promoter-mutated tumors showed longer progression-free survival 
Astrocytomas

Oligodendrogliomas Oligoastrocytomas
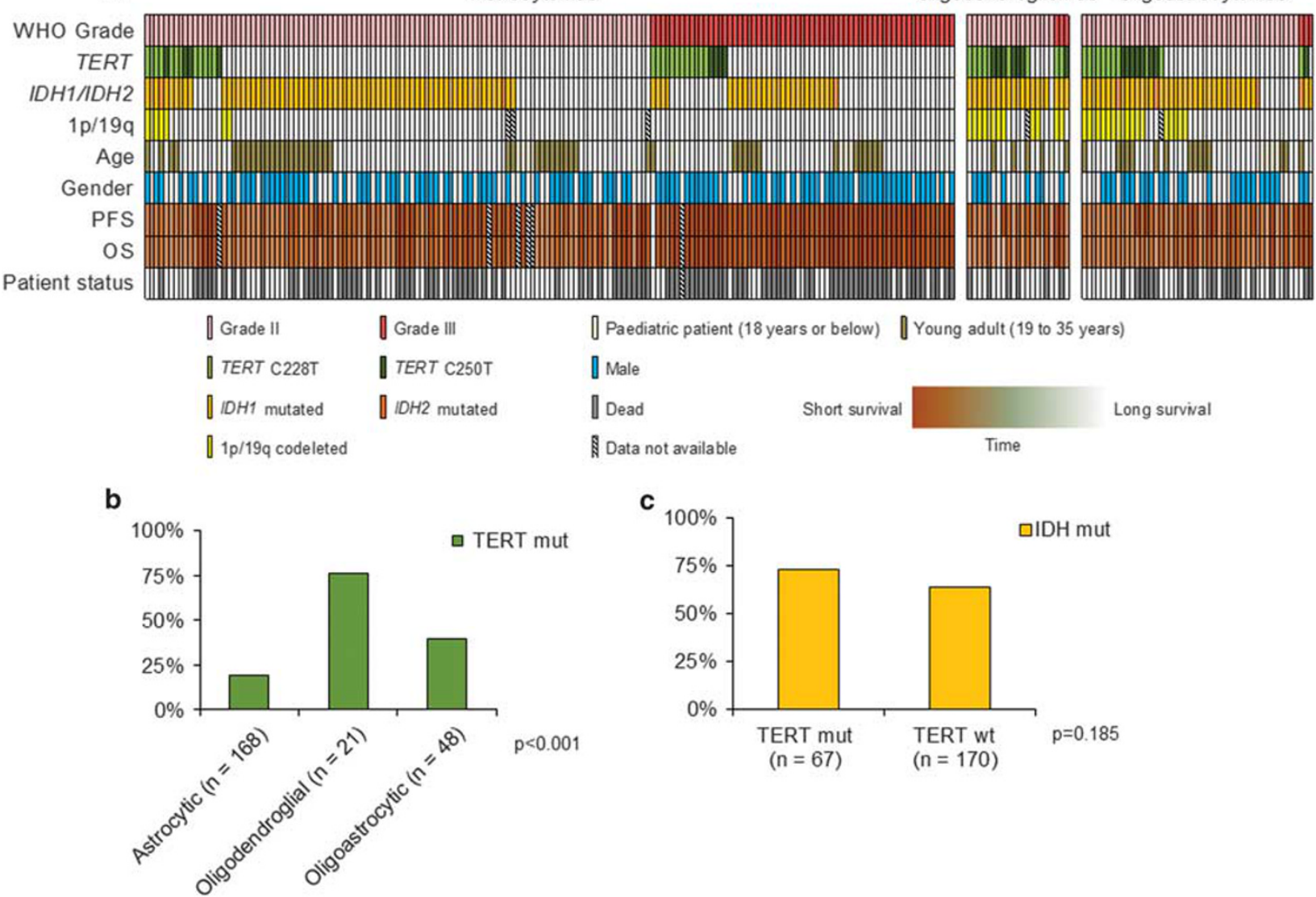

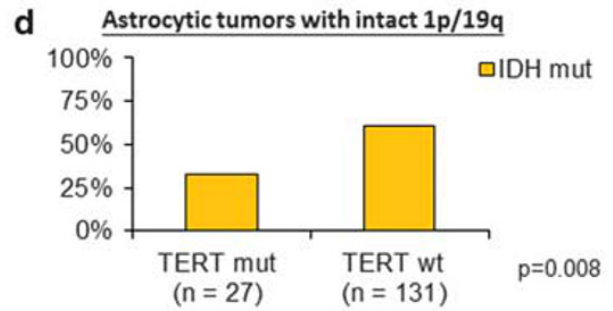

f

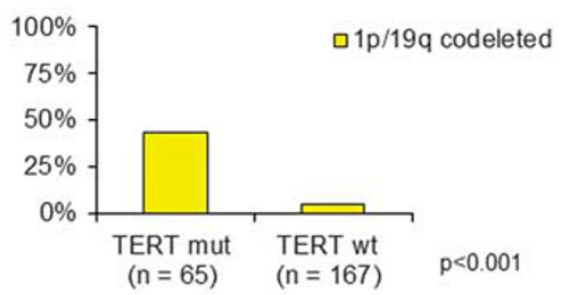

D Paediatric patient (18 years or below) \Young adult (19 to 35 years)

】Male

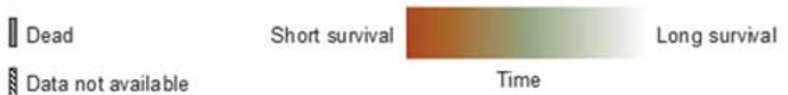

C

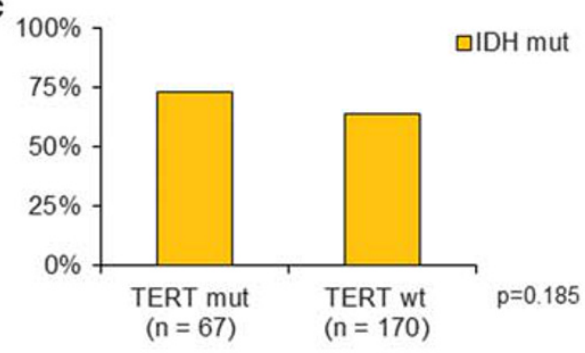

e Oligodendroglial tumors

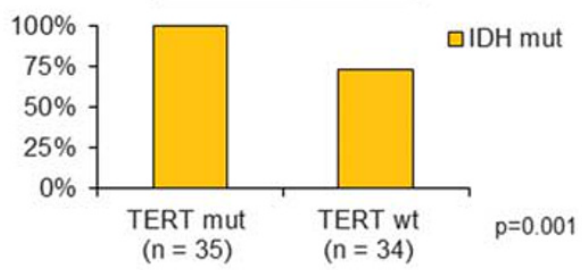

g

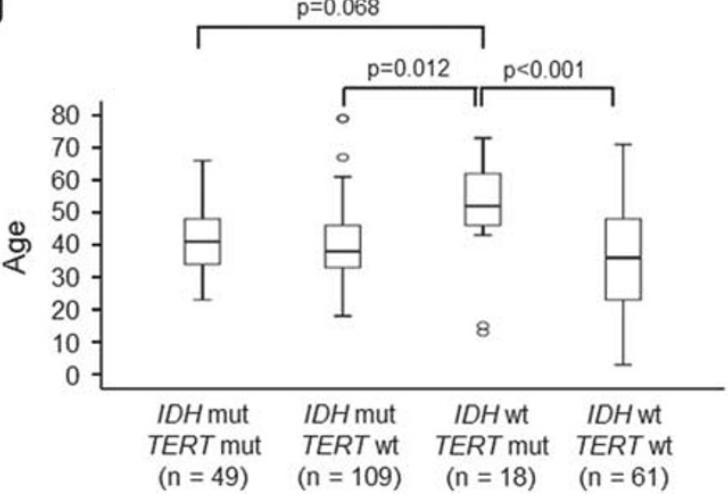

Figure 2 Correlations between clinicopathological and molecular variables of lower-grade gliomas. Clinical and molecular data of 237 lower-grade gliomas (a). TERT promoter mutation is associated with oligodendroglial histology $\left(P<0.001, \chi^{2}\right.$-test) (b). TERT promoter mutation was not associated with IDH mutation in the whole cohort $\left(P=0.185, \chi^{2}\right.$-test) (c). TERT promoter mutation was inversely associated with $I D H$ mutation among astrocytic tumors with intact $1 \mathrm{p} / 19 \mathrm{q}\left(P=0.008, \chi^{2}\right.$-test) (d), co-occurred with $I D H$ mutation among oligodendroglial tumors $(P=0.001$, Fisher's exact test $)(\mathbf{e})$, and co-occurred with $1 \mathrm{p} / 19 \mathrm{q}$ codeletion in the whole cohort $\left(P<0.001, \chi^{2}\right.$-test $)$ (f). Patients with $I D H$ wild-type-TERT-mutated gliomas are older than those with $I D H$ wild-type-TERT wild-type tumors, IDH-mutatedTERT-wild-type tumors and trended to be older than IDH-mutated-TERT-mutated tumors, (One-way ANOVA) (g). Mut, mutated; wt, wild type. 
Table 2 Univariate analysis of clinical and molecular variables

\begin{tabular}{|c|c|c|c|c|c|}
\hline Variables & $\mathrm{N}$ & $\begin{array}{l}\text { Median PFS } \\
\text { (months) }\end{array}$ & $\mathrm{P}$ & $\begin{array}{l}\text { Median OS } \\
\text { (months) }\end{array}$ & $\mathrm{P}$ \\
\hline \multicolumn{6}{|l|}{ Age } \\
\hline$\leq 35$ years & 81 & 78 & 0.02 & 141.5 & 0.005 \\
\hline$>35$ years & 150 & 48 & & 65.6 & \\
\hline \multicolumn{6}{|l|}{ WHO Grade } \\
\hline Grade II & 163 & 84 & $<0.001$ & 122.5 & $<0.001$ \\
\hline Grade III & 68 & 19.6 & & 24 & \\
\hline \multicolumn{6}{|l|}{ Histology } \\
\hline Astrocytic & 162 & 47 & 0.006 & 59.1 & $<0.001$ \\
\hline Oligodendroglial & 21 & 108 & & NR & \\
\hline Oligoastrocytic & 48 & 108.4 & & 120.6 & \\
\hline \multicolumn{6}{|c|}{ TERT promoter mutation } \\
\hline Mutant & 65 & 108 & 0.103 & 126 & 0.037 \\
\hline Wild type & 166 & 48 & & 67 & \\
\hline \multicolumn{6}{|l|}{ IDH mutation } \\
\hline Mutant & 157 & 71 & $<0.001$ & 120 & $<0.001$ \\
\hline Wild-type & 74 & 20 & & 24 & \\
\hline \multicolumn{6}{|l|}{$1 p / 19 q$ codeletion } \\
\hline Yes & 37 & NR & $<0.001$ & 142.1 & $<0.001$ \\
\hline No & 189 & 48 & & 60 & \\
\hline
\end{tabular}

Abbreviations: N, number of case; NR, median survival not yet reached; OS, overall survival; PFS, progression-free survival.

$(P=0.001)$ and longer OS $(P<0.001)$. As TERT promoter mutation was highly associated with $1 \mathrm{p} /$ 19q codeletion, which may account for the favorable prognostic effect, we evaluated the subset of 116 $I D H$-mutated tumors lacking $1 \mathrm{p} / 19 \mathrm{q}$ codeletion and found that 19 TERT promoter-mutated tumors exhibited favorable PFS $(P=0.027)$ and OS $(P=0.004)$. Consistent with this observation, subset analysis in $97 \mathrm{IDH}$-mutated astrocytomas also revealed that TERT promoter mutation in 14 astrocytomas was associated with good prognosis in both PFS $(P=0.001)$ and OS $(P=0.001)$. In contrast, among the subset of $74 \mathrm{IDH}$ wild-type lower-grade gliomas, TERT promoter mutation was associated with shorter PFS $(P=0.001)$ and OS $(P=0.001)$. In the subset of $65 \mathrm{IDH}$ wild-type astrocytomas, 16 TERT promoter-mutated astrocytomas exhibited unfavorable PFS $(P=0.007)$ and OS $(P=0.008)$.

Multivariate analysis was performed by Cox proportional hazards model to evaluate the independent prognostic values of the clinical and molecular variables (Table 4). Variables evaluated in multivariate analysis included age, WHO grade, tumor histology, TERT promoter mutation, IDH mutation, and $1 \mathrm{p} / 19 \mathrm{q}$ codeletion. Age $\leq 35$ years $(P=0.001)$, WHO grade II $(P<0.001)$, IDH mutation $(P=0.013)$ and $1 \mathrm{p} / 19 \mathrm{q}$ codeletion $(P=0.036)$ were favorable prognostic factors for PFS. Age $\leq 35$ years $(P=0.001)$, WHO grade II $(P<0.001)$, and IDH mutation $(P=0.009)$ were favorable prognostic factors for OS. 1p/19q codeletion showed a strong trend as good prognostic factor for OS $(P=0.067)$. As TERT promoter mutation showed opposite prognostic value in $I D H$-mutated and $I D H$ wild-type tumors in univariate analysis, multivariate analysis was conducted separately in the two subsets to further define the prognostic implication of TERT promoter mutation. Among IDH-mutated tumors, Age $\leq 35$ years $(P=0.015)$, WHO grade II $(P=0.001)$, TERT promoter mutation $(P=0.007)$, and $1 \mathrm{p} / 19 \mathrm{q}$ codeletion $(P=0.047)$ were favorable prognostic factors for PFS. Age $\leq 35$ years $(P=0.013)$, WHO grade II $(P<0.001)$, and TERT promoter mutation $(P=0.002)$ were good prognostic factors for OS. $1 \mathrm{p} /$ $19 \mathrm{q}$ codeletion showed a strong trend as good prognostic factor for OS $(P=0.057)$. Among $I D H$ wild-type tumors, WHO grade II was a favorable prognostic factor for PFS $(P=0.002)$ and OS $(P<0.001)$. TERT promoter mutation exhibited as a poor prognostic factor for PFS $(P=0.027)$ and a trend as a poor prognostic factor for OS $(P=0.07)$.

\section{Discussion}

Our study evaluated the frequency of TERT promoter mutation and its clinical significance in lowergrade gliomas. We demonstrated that TERT promoter mutation was frequently detected in lower-grade gliomas, particularly in oligodendroglial tumors. The strong association of TERT promoter mutation with oligodendroglial histology and $1 p / 19 q$ codeletion suggested that this activating mutation was involved in oligodendroglial oncogenesis, which was also demonstrated by other groups. ${ }^{15-17}$ Koelsche et $a 1^{17}$ analyzed over 1500 tumors of nervous system and found that TERT promoter mutation was inversely associated with $I D H$ mutation. Similar inverse correlation was shown by Nonoguchi et al ${ }^{18}$ examining over 350 glioblastomas. In our cohort of lower-grade gliomas, no correlation was found between IDH mutation and TERT promoter mutation. Interestingly, we observed opposite correlations between the two molecular markers in different subsets of tumors-inverse correlation in the subset of astrocytomas with intact $1 p / 19 q$ and co-occurring correlation in the subset of oligodendroglial tumors. These observations were consistent with the current literature ${ }^{15-18}$ and we speculated that the co-occurring association between $1 \mathrm{p} / 19 \mathrm{q}$ codeletion and $I D H$ mutation as well as $1 \mathrm{p} / 19 \mathrm{q}$ codeletion and TERT mutation 'neutralized' the inverse correlation between IDH mutation and TERT mutation in the whole cohort. Nevertheless, $I D H$ mutation and $1 \mathrm{p} / 19 \mathrm{q}$ codeletion have been suggested as early event in gliomagenesis. ${ }^{21,22}$ With the high frequency of TERT promoter mutation identified in oligodendrogliomas and its co-occurrence with $1 \mathrm{p} / 19 \mathrm{q}$ codeletion, it will be interesting to examine these molecular markers in paired primary and recurrent oligodendrogliomas to determine their temporal relationship. 
All lower grade gliomas $(n=231)$
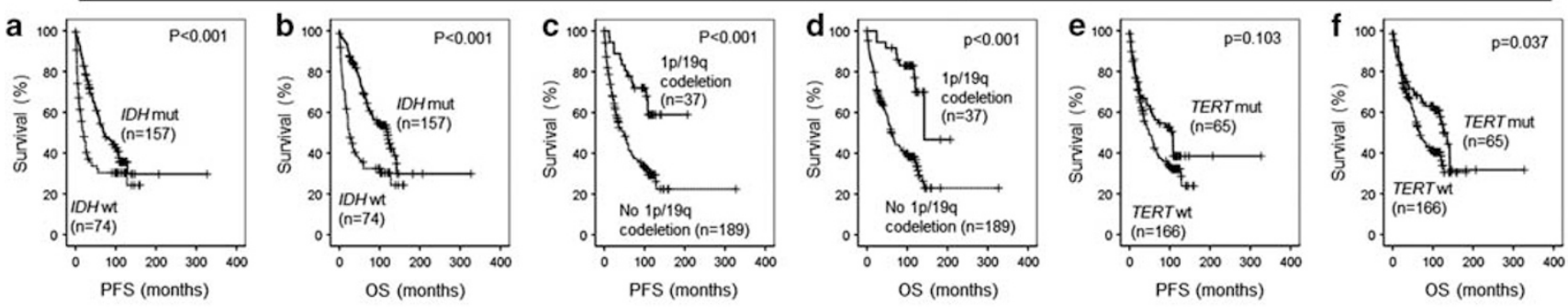

IDH mutated lower grade gliomas ( $n=157)$
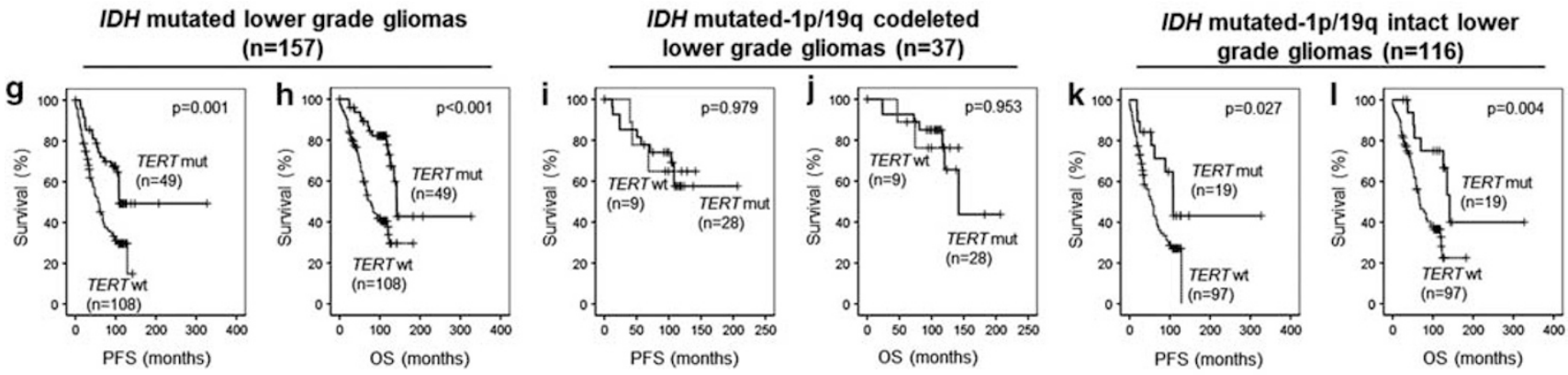

IDH mutated astrocytomas $(\mathrm{n}=97)$
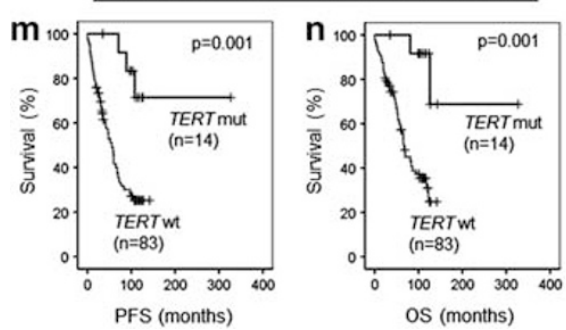

IDH wild type lower grade gliomas $(n=74)$
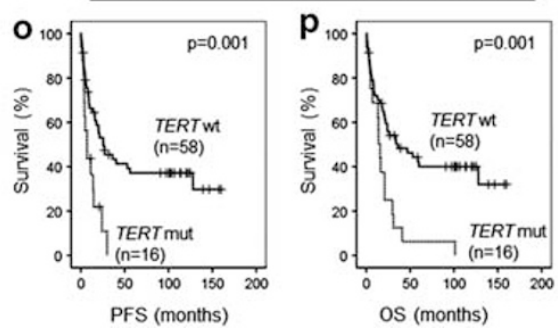

$I D H$ wild type astrocytomas $(n=65)$
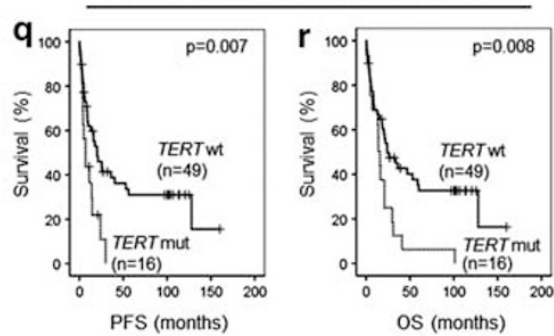

Figure 3 Kaplan-Meier survival analysis of $I D H$ mutation, 1p/19q codeletion and TERT promoter mutation in lower-grade gliomas. Among all lower-grade gliomas analyzed, IDH mutation was associated with longer PFS (a) and OS (b), 1p/19q codeletion was associated with longer PFS (c) and OS (d), TERT promoter mutation was associated with longer OS (f) but not PFS (e). Among all IDH-mutated tumors, TERT promoter mutation was associated with longer PFS (g) and OS (h). Among all $I D H$-mutated-1p/19q codeleted tumors, TERT promoter mutation was not associated with PFS (i) nor OS (j). Among all IDH-mutated-1p/19q intact tumors, TERT promoter mutation was associated with longer PFS (k) and OS (l). Among all IDH-mutated astrocytomas, TERT promoter mutation was associated with longer PFS (m) and OS (n). In IDH wild-type tumors, TERT promoter mutation was associated with shorter PFS (o) and OS (p). Among IDH wild-type astrocytomas, TERT promoter mutation was associated with shorter PFS (q) and OS (r).

Apart from the subgroup of lower-grade gliomas harboring all the three genetic alterations, there existed TERT promoter-mutated lower-grade gliomas lacking 1p/19q codeletion and $I D H$ mutation. Importantly, prognostic difference was found between these molecular subgroups. TERT promoter mutation had opposite prognostic values in $I D H$ mutated and $I D H$ wild-type tumors in both univariate analysis and multivariate analysis. In $I D H$ wildtype lower-grade gliomas, TERT promoter mutation identified aggressive tumors. TERT promoter mutation was frequently detected in primary glioblastoma lacking $I D H$ mutation and was associated with poor prognosis in glioblastoma patients. ${ }^{15,17}$ The IDH wild-type-TERT-mutated lower-grade gliomas identified in our series, with a similarly worsened outcome compared with the IDH-mutated lowergrade gliomas, may require more treatment and followup. In contrast, among $I D H$-mutated lower-grade gliomas, particularly in astrocytomas, TERT promoter mutation exhibited favorable prognosis. Existence of this good prognostic subgroup in $I D H$ mutated lower-grade gliomas was further evidenced by the association between TERT promoter mutation and good prognosis in $I D H$-mutated tumors lacking $1 \mathrm{p} / 19 \mathrm{q}$ codeletion as $1 \mathrm{p} / 19 \mathrm{q}$ codeletion is a group with good prognosis anyway. These findings suggested that TERT promoter mutation could potentially aid the molecular stratification of lower-grade gliomas in addition to $I D H$ mutation and $1 \mathrm{p} / 19 \mathrm{q}$ codeletion, especially in patients with astrocytic tumors.

Contrary to the concept of promoter methylation leading to gene silencing, TERT promoter methylation was found to be associated with TERT expression in other cancers including pediatric brain tumors. $^{23-25}$ Together with the exceedingly low frequency of TERT promoter mutation identified in 
Table 3 Univariate analysis in different subsets of lower-grade gliomas

\begin{tabular}{|c|c|c|c|c|c|c|}
\hline Lower-grade glioma subset & TERT promoter & $\mathrm{N}$ & Median PFS (months) & $\mathrm{P}$ & Median OS (months) & $\mathrm{P}$ \\
\hline \multicolumn{7}{|l|}{ IDH mutated } \\
\hline & Mutated & 49 & 108.4 & 0.001 & 142.1 & $<0.001$ \\
\hline & Wild type & 108 & 58 & & 74.4 & \\
\hline \multicolumn{7}{|c|}{$I D H$-mutated-1p/19q codeleted } \\
\hline & Mutated & 28 & NR & 0.979 & 142.1 & 0.953 \\
\hline & Wild type & 9 & NR & & NR & \\
\hline \multicolumn{7}{|l|}{$I D H$-mutated-1p/19q intact } \\
\hline & Mutated & 19 & 108.4 & 0.027 & 141.5 & 0.004 \\
\hline & Wild type & 97 & 56 & & 67 & \\
\hline \multicolumn{7}{|l|}{ IDH-mutated astrocytomas } \\
\hline & Mutated & 14 & NR & 0.001 & NR & 0.001 \\
\hline & Wild type & 83 & 56 & & 67 & \\
\hline \multicolumn{7}{|l|}{ IDH wild type } \\
\hline & Mutated & 16 & 7 & 0.001 & 14.1 & 0.001 \\
\hline & Wild type & 58 & 25.6 & & 36 & \\
\hline \multicolumn{7}{|l|}{ IDH wild-type astrocytomas } \\
\hline & Mutated & 16 & 7 & 0.007 & 14.1 & 0.008 \\
\hline & Wild type & 49 & 20.3 & & 24 & \\
\hline
\end{tabular}

Abbreviations: N, number of case; NR, median survival not yet reached; OS, overall survival; PFS, progression-free survival.

Table 4 Multivariate analysis in different subsets of lower-grade gliomas

\begin{tabular}{|c|c|c|c|c|c|}
\hline \multirow{2}{*}{ Tumor subset } & \multirow{2}{*}{ Variables } & \multicolumn{2}{|l|}{ PFS } & \multicolumn{2}{|l|}{ OS } \\
\hline & & $H R(95 \% C I)^{\mathrm{a}}$ & $\mathrm{P}$ & $H R(95 \% C I)^{\mathrm{a}}$ & $\mathrm{P}$ \\
\hline \multirow[t]{7}{*}{ All lower-grade gliomas } & Age $\leq 35$ years & $0.54(0.37-0.79)$ & 0.001 & $0.52(0.34-0.78)$ & 0.001 \\
\hline & WHO grade II & $0.36(0.24-0.54)$ & $<0.001$ & $0.28(0.18-0.43)$ & $<0.001$ \\
\hline & Oligodendroglial & 1 & 0.991 & 0.54 & 0.183 \\
\hline & Oligoastrocytic & 0.87 & 0.579 & 0.82 & 0.464 \\
\hline & TERT promoter mutation & 0.91 & 0.695 & 0.83 & 0.433 \\
\hline & $I D H$ mutation & $0.63(0.43-0.91)$ & 0.013 & $0.6(0.41-0.88)$ & 0.009 \\
\hline & 1p/19q codeletion & $0.49(0.25-0.96)$ & 0.036 & 0.47 & 0.06 \\
\hline \multirow[t]{6}{*}{$I D H$-mutated lower-grade gliomas } & Age $\leq 35$ years & $0.55(0.34-0.89)$ & 0.015 & $0.51(0.3-0.87)$ & 0.013 \\
\hline & WHO grade II & $0.39(0.22-0.69)$ & 0.001 & $0.3(0.16-0.55)$ & $<0.001$ \\
\hline & Oligodendroglial & 1.6 & 0.215 & 0.8 & 0.65 \\
\hline & Oligoastrocytic & 1.13 & 0.675 & 1.15 & 0.645 \\
\hline & TERT promoter mutation & $0.44(0.24-0.8)$ & 0.007 & $0.34(0.18-0.66)$ & 0.002 \\
\hline & 1p/19q codeletion & $0.5(0.25-0.99)$ & 0.047 & 0.46 & 0.057 \\
\hline \multirow[t]{5}{*}{$I D H$ wild-type lower-grade gliomas } & Age $\leq 35$ years & 0.91 & 0.762 & 0.93 & 0.829 \\
\hline & WHO grade II & $0.36(0.19-0.69)$ & 0.002 & $0.29(0.15-0.58)$ & $<0.001$ \\
\hline & Oligodendroglial & $<0.001$ & 0.974 & $<0.001$ & 0.975 \\
\hline & Oligoastrocytic & 0.54 & 0.29 & 0.37 & 0.186 \\
\hline & TERT promoter mutation & $2.16(1.09-4.28)$ & 0.027 & 1.83 & 0.07 \\
\hline
\end{tabular}

Abbreviations: 95\%CI, 95\% confidence interval; HR, hazard ratio; OS, overall survival; PFS, progression-free survival.

${ }^{\mathrm{a}} 95 \%$ confidence intervals are shown for variables with significant $P$-value.

over 350 pediatric brain tumors by Koelsche et al, ${ }^{17}$ TERT promoter methylation probably represents a major mechanism for telomerase activation in pediatric brain tumors. Intriguingly, a recent study by Arita et $a 1^{26}$ demonstrated that TERT promoter mutation rather than methylation was the main mechanism for TERT upregulation in adult gliomas.
Such results not only illustrated the diverse mechanisms of telomerase activation in different cancer types, but also demonstrated the distinct pathogenesis between pediatric and adult brain tumors.

One of the crucial findings in this study was the opposite prognostic values of TERT promoter mutation in $I D H$-mutated and $I D H$ wild-type lower-grade 
gliomas. Interestingly, subgroup-specific prognostic implication of TERT promoter mutation was also demonstrated in medulloblastoma by Remke et $a l^{27}$, with the mutation identified patients with good prognosis in $\mathrm{SHH}$ subgroup and patients with bad prognosis in Group 4 subgroup. Such subgroupspecific prognostic values, together with its high frequency in certain groups of brain tumors and the relatively easy assay method (PCR followed by direct sequencing), made TERT promoter mutation as an emerging molecular marker for patient stratification in neuro-oncology.

In conclusion, TERT promoter is frequently mutated in lower-grade gliomas, with a particularly high incidence in oligodendroglial tumors. The mutations identify a favorable prognostic subset of $I D H$-mutated-1p/19q intact or astrocytic tumors and an aggressive subset of $I D H$ wild-type tumors. Our study suggests the potential values of TERT promoter mutational analysis in molecular classification and prognostic evaluation in lower-grade gliomas in the era of personalized medicine.

\section{Acknowledgments}

This study was supported by the National Science Foundation of China (grant no. 81172412).

\section{Disclosure/conflict of interest}

The authors declare no conflict of interest.

\section{References}

1 Louis DN, Ohgaki H, Wiestler OD, et al. WHO Classification of Tumours of the Central Nervous System. International Agency for Research on Cancer: Lyon; 2007, pp 14-67.

2 van den Bent MJ. Interobserver variation of the histopathological diagnosis in clinical trials on glioma: a clinician's perspective. Acta Neuropathol 2010;120: 297-304.

3 Reifenberger J, Reifenberger G, Liu L, et al. Molecular genetic analysis of oligodendroglial tumors shows preferential allelic deletions on $19 q$ and $1 p$. Am J Pathol 1994;145:1175-1190.

4 Smith JS, Perry A, Borell TJ, et al. Alterations of chromosome arms $1 p$ and $19 q$ as predictors of survival in oligodendrogliomas, astrocytomas, and mixed oligoastrocytomas. J Clin Oncol 2000;18:636-645.

5 van den Bent MJ, Brandes AA, Taphoorn MJ, et al. Adjuvant procarbazine, lomustine, and vincristine chemotherapy in newly diagnosed anaplastic oligodendroglioma: long-term follow-up of EORTC brain tumor group study 26951. J Clin Oncol 2013;31:344-350.

6 Cairncross G, Wang M, Shaw E, et al. Phase III trial of chemoradiotherapy for anaplastic oligodendroglioma: long-term results of RTOG 9402. J Clin Oncol 2013;31: 337-343.

7 van den Bent MJ, Reni M, Gatta G, et al. Oligodendroglioma. Crit Rev Oncol Hematol 2008;66:262-272.
8 Parsons DW, Jones S, Zhang X, et al. An integrated genomic analysis of human glioblastoma multiforme. Science 2008;321:1807-1812.

9 Camelo-Piragua S, Jansen M, Ganguly A, et al. Mutant IDH1-specific immunohistochemistry distinguishes diffuse astrocytoma from astrocytosis. Acta Neuropathol 2010;119:509-511.

10 Capper D, Reuss D, Schittenhelm J, et al. Mutationspecific IDH1 antibody differentiates oligodendrogliomas and oligoastrocytomas from other brain tumors with oligodendroglioma-like morphology. Acta Neuropathol 2011;121:241-252.

11 Yan H, Parsons DW, Jin G, et al. IDH1 and IDH2 mutations in gliomas. N Engl J Med 2009;360: 765-773.

12 Metellus P, Coulibaly B, Colin C, et al. Absence of IDH mutation identifies a novel radiologic and molecular subtype of WHO grade II gliomas with dismal prognosis. Acta Neuropathol 2010;120:719-729.

13 Horn S, Figl A, Rachakonda PS, et al. TERT promoter mutations in familial and sporadic melanoma. Science 2013;339:959-961.

14 Huang FW, Hodis E, Xu MJ, et al. Highly recurrent TERT promoter mutations in human melanoma. Science 2013;339:957-959.

15 Killela PJ, Reitman ZJ, Jiao Y, et al. TERT promoter mutations occur frequently in gliomas and a subset of tumors derived from cells with low rates of selfrenewal. Proc Natl Acad Sci USA 2013;110:6021-6026.

16 Arita H, Narita Y, Fukushima S, et al. Upregulating mutations in the TERT promoter commonly occur in adult malignant gliomas and are strongly associated with total 1p19q loss. Acta Neuropathol 2013;126: 267-276.

17 Koelsche C, Sahm F, Capper D, et al. Distribution of TERT promoter mutations in pediatric and adult tumors of the nervous system. Acta Neuropathol 2013;126:907-915.

18 Nonoguchi N, Ohta T, Oh JE, et al. TERT promoter mutations in primary and secondary glioblastomas. Acta Neuropathol 2013;126:931-937.

19 Chan AK, Pang JC, Chung NY, et al. Loss of CIC and FUBP1 expressions are potential markers of shorter time to recurrence in oligodendroglial tumors. Mod Pathol 2014;27:332-342.

20 Dong Z, Pang JS, Ng MH, et al. Identification of two contiguous minimally deleted regions on chromosome 1p36.31-p36.32 in oligodendroglial tumours. $\mathrm{Br} \mathrm{J}$ Cancer 2004;91:1105-1111.

21 Watanabe T, Nobusawa S, Kleihues $\mathrm{P}$, et al. IDH1 mutations are early events in the development of astrocytomas and oligodendrogliomas. Am J Pathol 2009;174:1149-1153.

22 Cairncross G, Jenkins R. Gliomas with 1p/19q codeletion: a.k.a. oligodendroglioma. Cancer J 2008;14: 352-357.

23 Castelo-Branco P, Choufani S, Mack S, et al. Methylation of the TERT promoter and risk stratification of childhood brain tumours: an integrative genomic and molecular study. Lancet Oncol 2013;14:534-542.

24 de Wilde J, Kooter JM, Overmeer RM, et al. hTERT promoter activity and CpG methylation in HPVinduced carcinogenesis. BMC Cancer 2010;10:271.

25 Guilleret I, Yan P, Grange F, et al. Hypermethylation of the human telomerase catalytic subunit (hTERT) gene correlates with telomerase activity. Int J Cancer 2002; 101:335-341. 
26 Arita H, Narita Y, Takami H, et al. TERT promoter mutations rather than methylation are the main mechanism for TERT upregulation in adult gliomas. Acta Neuropathol 2013;126:939-941.
27 Remke M, Ramaswamy V, Peacock J, et al. TERT promoter mutations are highly recurrent in $\mathrm{SHH}$ subgroup medulloblastoma. Acta Neuropathol 2013; 126:917-929.

Supplementary Information accompanies the paper on Modern Pathology website (http://www.nature.com/ modpathol) 